\title{
Colour Coding of Information Layers in Computer Displays
}

\author{
Darren Van Laar ${ }^{*}$, Terry Williams ${ }^{*}$, Ian Umbers ${ }^{\dagger} \&$ Steve Smeaton $^{\dagger}$ \\ *Department of Psychology \\ University of Portsmouth \\ Portsmouth P01 2ER \\ UNITED KINGDOM \\ vanlaard@psyc.port.ac.uk \\ †Computing Systems and MMI Group \\ Nuclear Electric PLC \\ Barnett Way, Barnwood \\ Gloucester GL4 7RS \\ UNITED KINGDOM
}

\begin{abstract}
A method is reported which enables designers to analyse computer displayed information into a task hierarchy, and then allocate sets of colour codes to reflect this information structure. These sets of colours are derived from principles of cartography and colour science, and give rise to the impression of layers of information, where elements of the display most relevant to the task appears more conspicuous and nearer to the user than less task relevant information. The application of this method to typical control room displays is described.
\end{abstract}

KEYWORDS interface design, cartography, colour coding, colour displays, design

\section{INTRODUCTION}

The nature of good interface design lies in the effective communication of information that is directly relevant to the completion of a user's task. Previous work in cartography has advocated the use of conceptual layers as a means to organise task relevant data elements on a map or display (Wood, 1968).

Although such an approach has proved its worth in cartography, much cartographic design pays little attention to principles of colour science and often leads to inadequate map design (Taylor, 1984). More recently, this approach has been applied to computer based air-traffic control displays with good initial results (Reynolds, 1994). Besides maps or schematic representations it may be possible to use colour science based cartographic principles to design alphanumeric, tabular or graphical displays of the type typically found in industrial or emergency control rooms.

This paper offers a method of producing hierarchical visual effects on control room computer displays. A set of palettes for producing the perceptual effect of layers of information has been identified, and a method of application developed.

\section{AIMS AND OBJECTIVES}

Nuclear power station control room displays in the UK are predominately monochrome, green on black or yellow on black. A methodology for colour coding using 24-bit colour displays was required by the Nuclear Industry with the following objectives:

- To provide the designer with a set of colours with which to code display formats according to current psychological principles and standards governing the perception of colour from emissive displays.

- Such colour sets should offer a way of visually emphasizing task relevant information.

- The entire set of colours should be dividable into smaller sets that will correspond to a series of information layers allocated by the designer according to task relevance. 
- The set should avoid the pitfalls associated with the application of colour to displays. Such factors include colour contrast effects, visual after effects, ambiguities, and unwanted chromostereopsis.

- The colour sets should be applicable across the range of different power station displays so as to maintain consistency.

- No significant alterations were to be made to the content and structure of existing displays.

\section{DERIVATION OF COLOUR SETS}

The effect of information layers on displays is intended to be similar to that within road maps, where more important information (e.g. main roads, motorways), appear more conspicuous and immediate than less important information (e.g. land use, contours), whilst maintaining the visibility and discriminability of all information.

Six 'palettes' of colours, one achromatic set, four layering sets, and an alarm set were developed according to principles of colour science rather than employing graphic designers. Each layering palette consisted of six colours, a grey and five colours which differed in hue, but which had the same level of lightness and saturation. As long as they are far apart in colour space, the actual hues of the five colours are unimportant, but have been selected to be a red, a brown, a green, a blue and a purple in the current version of the methodology.

Palettes differ in lightness and saturation between layers, but contain colours which have the same five hues. This enables conceptually similar elements to be grouped between layers. In this way, for example, related information of different levels of importance about the same power station area such as boiler area and boiler vessel, could be coded by different levels of green.

Using task analytical techniques, elements of the display are first categorized into one of six information layers, Layer One containing the least important elements of the display, and Layer Six containing display elements most relevant to the task. Colours are then allocated to each of the layers.

Layer One is the background layer, on which all other layers will be seen to rest. Besides the background colour, screen and display boundary lines, labels describing the display also appear on this layer. To further de-emphasise this information, all elements on this layer are from the achromatic palette. Layer Two contains low level task grouping areas, whereby regions of the display are grouped by background colour or 'wash'. Where mimic displays are required such as schematic boilers or turbines, these items also appear on Layer Two. Layer Three contains titles to areas of the display. These need to appear as more important than the background, but less important than other text. Level Four contains static reference text, such as the units in which temperature or pressure are measured, and similar labels. Level Five appears to lie upon all the previous levels and contains frequently updated information such as changing pressure values or graphics. A final, 'alarm' layer, Layer Six, shows infrequent but highly relevant information which needs to appear as immediately conspicuous and important. The colour coding of this layer is based on different principles to the other layers, and contains highly saturated colours chosen according to industry standards and conventions (e.g. bright red, yellow, orange).

The methodology described above has been applied successfully to a large number of control room displays, and has substantially improved the appearance and conspicuity of task relevant information. Prediction of the exact colours required produce the layering effect has been implemented in a spreadsheet. Experiments quantifying these improvements are currently being planned.

\section{REFERENCES}

Reynolds, L. (1994) Colour for air traffic control displays. Displays, 15, 215-225.

Taylor, S. (1984) Colour design in aviation cartography. Displays, 10, 187-194.

Wood, M. (1968). Visual Perception and map design. Cartographic Journal, 5, 54-64. 